Algebraic $\&$ Geometric $\mathcal{T}$ opology

Volume 4 (2004) 943-960

Published: 22 October 2004

ATG

\title{
Partition complexes, duality and integral tree representations
}

\author{
Alan Robinson
}

\begin{abstract}
We show that the poset of non-trivial partitions of $\{1,2, \ldots, n\}$ has a fundamental homology class with coefficients in a Lie superalgebra. Homological duality then rapidly yields a range of known results concerning the integral representations of the symmetric groups $\Sigma_{n}$ and $\Sigma_{n+1}$ on the homology and cohomology of this partially-ordered set.
\end{abstract}

AMS Classification 05E25; 17B60, 55P91

Keywords Partition complex, Lie superalgebra

\section{Introduction}

This paper reveals the geometry underlying certain integral representations of the symmetric groups. It claims few original results, but gives a unified geometrical and homological treatment and frequently strengthens the known theorems. Above all it aims to show that topological methods give efficient proofs of many of them.

Geometric realization converts the lattice of non-trivial partitions of a set with $n$ elements into the space of fully-grown (that is, non-degenerate) trees with leaves labelled by the set $\{0,1, \ldots, n\}$. This space has reduced homology in degree $n-3$ only. Its integral cohomology is a representation of $\Sigma_{n}$ which is isomorphic to the Lie representation $\mathrm{Lie}_{n}$, up to a twist by the sign character. It follows from the $\Sigma_{n+1}$-symmetry of the tree space that $\mathrm{Lie}_{n}$ extends to an integral representation of $\Sigma_{n+1}$.

Many mathematicians have contributed to the identification of the cohomology of the partition lattice with the twisted Lie representation: excellent summaries of the history of the theory are given in [6] and [12]. We show that the isomorphism arises at the cocycle level from a duality structure: the partition lattice has a fundamental homology cycle with coefficients in a certain $\Sigma_{n}$-module $\mathfrak{S}_{n}$, 
and the isomorphism is induced by cap product. The module $\mathfrak{S}_{n}$ is the multilinear part of a free Lie superalgebra on $n$ generators of odd degree: it is isomorphic to the twisted Lie representation, though not uniquely so.

In the final section we give a geometrical derivation of a short exact sequence relating these representations, due to $\mathrm{S}$. Whitehouse [13].

The tree spaces are related to configuration spaces, and the occurrence of the Lie representations in this topological situation was observed by F. R. Cohen [3], 44].

\section{The space of fully-grown $n$-trees}

From [8] we recall topological properties of the space of $n$-trees, and prove a homeomorphism with the nerve of the lattice of partitions of the set $\{1,2, \ldots, n\}$.

A tree is a contractible 1-dimensional polyhedron $X$, and we shall always require $X$ to be compact. There is a unique coarsest triangulation of $X$, in which no vertex lies on exactly two edges. A vertex meeting more than two edges is called a node; a vertex incident upon only one edge is a free vertex.

An edge which connects two nodes is an internal edge. An edge with a free vertex is called a leaf.

Following [1, 2], we introduce a moduli space of trees with a fixed number of leaves.

Definition 2.1 An $n$-tree is a tree with the following extra structure.

(1) Every internal edge $e$ is assigned a length $l_{e}$ where $0<l_{e} \leq 1$. All leaves are conventionally assigned a length of 1 .

(2) The free vertices are labelled by a bijective correspondence with the set $\{0,1, \ldots, n\}$.

If $n \geq 2$, then the labelling of free vertices is equivalent to a labelling of the leaves by a bijection with the same set $\{0,1, \ldots, n\}$. The leaf labelled 0 is also called the root. Two $n$-trees are regarded as equivalent if there is an isometry between them which preserves the labelling. A tree is fully grown if at least one internal edge has length 1.

\section{Example 2.2 : the vertices $v_{A}$ of $T_{n}$}

Let $A$ be any subset of the set $[n]=\{0,1, \ldots, n\}$ of labels, such that $A$ and its complement $[n] \backslash A$ each have more than one element. 
We define $v_{A}$ to be the fully grown $n$-tree which has just one internal edge $e_{A}$ (which necessarily has length 1 ), and which has the leaves labelled by elements of $A$ attached at one end of this, and the leaves labelled by the elements of $[n] \backslash A$ at the other:

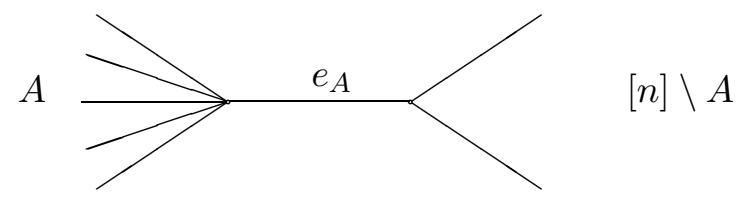

Since trees are regarded as the same when they are related by a label-preserving homeomorphism, it follows that $v_{A}=v_{[n] \backslash A}$.

In the next proposition $\Sigma_{n+1}$ denotes the set of all permutations of $\{0,1, \ldots, n\}$, and $\Sigma_{n}$ the subgroup leaving 0 fixed.

\section{Proposition 2.3}

(1) The space $T_{n}$ of all fully-grown $n$-trees is a simplicial complex of dimension $n-3$, in which the vertices are the trees $v_{A}$ of 2.2 .

(2) The space $\tilde{T}_{n}$ of all $n$-trees is the cone on $T_{n}$.

(3) The symmetric group $\Sigma_{n+1}$ acts on the right upon the pair $\left(\tilde{T}_{n}, T_{n}\right)$ by acting on the left upon the set of leaf labels. This action is simplicial.

Proof The proof is given in 8 8 . Suffice it to say here that $\tilde{T}_{n}$ has an evident cubical structure, with the internal edge-lengths as cubical coordinates. On the subspace $T_{n}$ the greatest edge-length is 1 , so there is a simplicial structure with barycentric coordinates proportional to the edge-lengths: the vertices are the $v_{A}$ of 2.2. and each $k$-simplex is an amalgam of $k+1$ of the original cubes as illustrated for $k=2$ on the left in the following diagram.
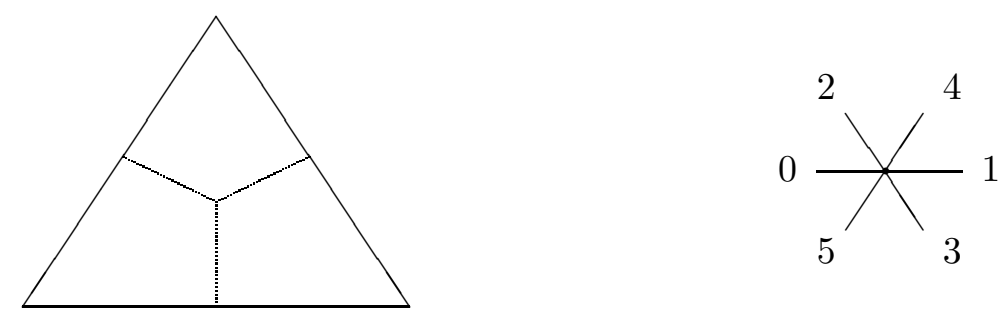
The space $\tilde{T}_{n}$ is evidently a cone with $T_{n}$ as its base. Its apex is a star-tree as illustrated on the right above, and the cone parameter maps a tree to its maximal internal edge-length. Therefore $\tilde{T}_{n}$ can be triangulated as the cone on the simplicial complex $T_{n}$. As with $T_{n}$, the original cubical structure is a subdivision of this triangulation.

From now on, we use the triangulations just constructed, unless the contrary is mentioned.

Lemma 2.4 Every $(n-4)$-simplex of $T_{n}$ is a face of exactly three topdimensional simplices.

Proof The top-dimensional simplices correspond to binary trees in which each node is the meet of three edges. An $(n-4)$-simplex of $T_{n}$ arises from a tree with one exceptional node of order four: it faces three top-dimensional simplices corresponding to the three ways of resolving this node into two nodes of order three [8].

Lemma 2.4 shows that $T_{n}$ has three-to-one incidence on codimension-one simplexes. As is well known, a triangulated closed manifold has two-to-one incidence, and consequently has a fundamental top-dimensional homology class (with integer coefficients, possibly twisted). We shall see in $\S_{4}$ that $T_{n}$ too has a fundamental homology class for which the coefficients are a certain module $\mathfrak{S}_{n}$.

Theorem 2.5 The space $T_{n}$ of fully-grown $n$-trees has the homotopy type of a wedge of $(n-1)$ ! spheres of dimension $n-3$. The $\Sigma_{n}$-module $\tilde{H}_{n-3}\left(T_{n}\right)$ restricts to the regular integral representation of the subgroup $\Sigma_{n-1}$.

Proof It is shown in [8] that $T_{n}$ is obtained from a contractible space by attaching $(n-1)$ ! simplices of dimension $n-3$ along their boundaries. These simplices correspond to the trees

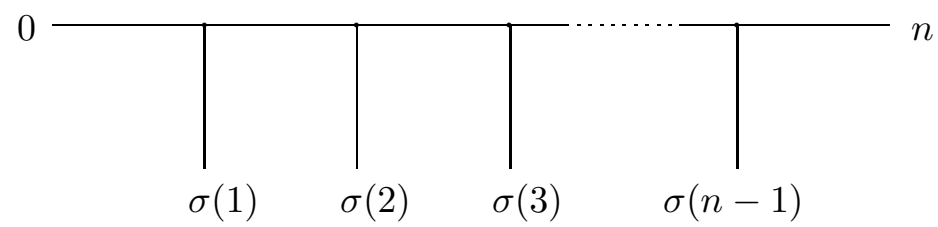

where $\sigma$ is any element of the permutation group $\Sigma_{n-1}$. Thus $T_{n}$ has the homotopy type of a wedge of spheres, as claimed; and the homology classes of these spheres are regularly permuted by the subgroup $\Sigma_{n-1}$. 


\subsection{Homeomorphism with the nerve of the partition lattice $\Lambda_{n}$}

We call a partition of the set $\{1,2, \ldots, n\}$ non-trivial if it is neither indiscrete nor discrete: that is, the number of equivalence classes is greater than 1 but less than $n$. The non-trivial partitions, ordered by refinement, form a lattice. We use the notation $\Lambda_{n}$ both for this lattice and for the simplicial complex which is its nerve.

Proposition 2.7 The nerve $\Lambda_{n}$ of the partition lattice is $\Sigma_{n}$-equivariantly homeomorphic to the space $T_{n}$ of fully-grown $n$-trees.

Proof We describe an explicit homeomorphism $\varphi: T_{n} \rightarrow \Lambda_{n}$. A point $\alpha \in T_{n}$ is an $n$-tree in which each internal edge has a given length between 0 and 1 .

For each $1 \leq i \leq n$ there is a unique arc $\gamma_{i}$ in the tree $\alpha$ which starts at the root labelled 0 and ends at the leaf labelled $i$. We parametrize all these arcs with unit speed, and we extend $\gamma_{i}$ to a map $[0, \infty) \rightarrow \alpha$ by setting $\gamma_{i}(t)$ to be constant at the vertex labelled $i$ for all $t \gg 0$.

As time increases, these arcs diverge and determine finer and finer partitions of $\{1,2, \ldots, n\}$. For every $t>0$ we define $\Pi_{\alpha}(t)$ to be the partition of $\{1,2, \ldots, n\}$ given by the equivalence relation

$$
i \sim j \quad \Longleftrightarrow \quad \gamma_{i}(t)=\gamma_{j}(t) .
$$

The partition $\Pi_{\alpha}(t)$ refines $\Pi_{\alpha}(s)$ if $t>s$. Further, $\Pi_{\alpha}(t)$ is discrete for all sufficiently large $t$; and it is indiscrete when $t$ is sufficiently close to zero. Hence there is a unique affine map $\rho:[0,1] \rightarrow[0, \infty)$ such that $\Pi_{\alpha}(\rho(t))$ is non-trivial exactly for $0<t<1$.

We can now define the point $\varphi(\alpha)$ in the nerve $\Lambda_{n}$ of the partition lattice, as follows. The barycentric coordinate of $\varphi(\alpha)$ with respect to any non-trivial partition $\Pi$ is to be the length of the interval $\left\{t \in[0,1] \mid \Pi_{\alpha}(\rho(t))=\Pi\right\}$. The map $\varphi: T_{n} \rightarrow \Lambda_{n}$ so defined is a homeomorphism because the fully-grown tree $\alpha$ is uniquely determined by its one-parameter family of partitions $\Pi_{\alpha}$. The map is clearly equivariant.

Corollary 2.8 The right action of $\Sigma_{n}$ on the nerve of the partition lattice $\Lambda_{n}$ extends to a right action of $\Sigma_{n+1}$.

Proof This is true of the action on $T_{n}$, since the action of $\Sigma_{n}$ there is the restriction of the action of the group $\Sigma_{n+1}$ of permutations of the full set $\{0,1, \ldots, n\}$ of labels. 


\section{The representation $\mathfrak{S}_{n}$ and the fundamental cycle}

\subsection{The Lie representations}

Let $\mathcal{L}_{n}$ be the free Lie ring on the set of generators $\left\{x_{i}\right\}_{1 \leq i \leq n}$. We denote by Lie $_{n}$ the $n$-linear part of $\mathcal{L}_{n}$. This can be described in many different ways. First, it is the direct summand of $\mathcal{L}_{n}$ spanned by all Lie monomials containing each of the $n$ generators exactly once. Second, it is isomorphic to the module of all natural transformations $\Phi^{\otimes n} \rightarrow \Phi$, where $\Phi$ is the forgetful functor from Lie rings to abelian groups. Third, it is the $n$th module in the Lie operad.

It is a standard exercise in the use of the Jacobi identity to show that $\mathrm{Lie}_{n}$ is a free abelian group of rank $(n-1)$ !, and that the left-regulated Lie brackets

$$
\lambda_{\sigma}=\left[x_{\sigma(1)},\left[x_{\sigma(2)},\left[\ldots\left[x_{\sigma(n-1)}, x_{n}\right] . .\right]\right]\right]
$$

for $\sigma \in \Sigma_{n-1}$ form a $\mathbb{Z}$-basis for $\operatorname{Lie}_{n}$ (see for example [7] or [13]). The symmetric group $\Sigma_{n}$ acts on $\mathrm{Lie}_{n}$ on the left by permuting the $n$ generators. The (integral) representation thus afforded is known as the Lie representation. The subgroup $\Sigma_{n-1}$ permutes the above standard basis simply and transitively. Thus the restricted representation $\operatorname{Res}_{\Sigma_{n-1}}^{\Sigma_{n}} \operatorname{Lie}_{n}$ is the regular integral representation of $\Sigma_{n-1}$.

We denote by $\operatorname{Lie}_{n}^{*}$ the dual representation $\operatorname{Hom}\left(\operatorname{Lie}_{n}, \mathbb{Z}\right)$. This has the same character as $\mathrm{Lie}_{n}$, and therefore $\mathrm{Lie}_{n}$ and $\mathrm{Lie}_{n}^{*}$ are isomorphic over the rationals. Over the integers, the two are distinct.

\section{Example 3.2}

Take $n=3$, and consider the essentially unique $\Sigma_{3}$-invariant Euclidean metric on the two-dimensional space $\mathrm{Lie}_{3} \otimes \mathbb{R}$. Both $\mathrm{Lie}_{3}$ and $\mathrm{Lie}_{3}^{*}$ are hexagonal lattices in this (self-dual) Euclidean space. However, in $\mathrm{Lie}_{3}$ the shortest vectors have the form $\left[x_{i},\left[x_{j}, x_{k}\right]\right]$, and so these are $(-1)$-eigenvectors of involutions $(j, k) \in \Sigma_{3}$. In $\operatorname{Lie}_{3}^{*}$ the shortest vectors are $(+1)$-eigenvectors of involutions.

Let $\varepsilon$ denote the sign character of $\Sigma_{n}$. Then we have the twisted module $\varepsilon \mathrm{Lie}_{n}$ which is the same abelian group $\operatorname{Lie}_{n}$ with the twisted action of $\Sigma_{n}$ defined by

$$
\sigma \cdot f\left(x_{1}, \ldots, x_{n}\right)=\varepsilon(\sigma) f\left(x_{\sigma(1)}, \ldots, x_{\sigma(n)}\right)
$$

for every multilinear Lie monomial $f$ and every $\sigma \in \Sigma_{n}$. We also denote the module $\varepsilon \mathrm{Lie}_{n}$ by $\varepsilon \otimes \mathrm{Lie}_{n}$ when we need to stress that it is the tensor product with the sign module. We similarly have the twisted representation $\varepsilon \mathrm{Lie}_{n}^{*}$, defined analogously. 


\subsection{Connection with Lie superrings}

There is a reinterpretation of the module $\varepsilon \operatorname{Lie}_{n}$ which significantly simplifies our theory. In place of the free Lie ring on the set of generators $\left\{x_{i}\right\}_{1 \leq i \leq n}$ we may consider the free Lie superring (or Lie superalgebra over $\mathbb{Z}$ ) having the $x_{i}$ as odd-degree generators. This we denote by $\mathcal{S}_{n}$. It is a graded algebra $\mathcal{S}_{n}^{\text {even }} \oplus \mathcal{S}_{n}^{\text {odd }}$ freely generated by the $x_{i}$ subject to the relations

$$
\begin{aligned}
{[a, b] } & =(-1)^{|a||b|+1}[b, a] \\
{[[a, b], c] } & =[a,[b, c]]-(-1)^{|a||b|}[b,[a, c]]
\end{aligned}
$$

when $a$ and $b$ are homogeneous elements of degrees $|a|$ and $|b|$ respectively.

Let $\mathfrak{S}_{n}$ denote the super-Lie representation of $\Sigma_{n}$. This we define to be the $n$-linear part of $\mathcal{S}_{n}$ : it is spanned by the super-Lie monomials containing each $x_{i}$ exactly once, and is a subgroup of $\mathcal{S}_{n}^{\text {even }}$ or $\mathcal{S}_{n}^{\text {odd }}$ according to the parity of $n$. The symmetric group acts by permuting the generators, exactly as in the Lie representation. We now determine the structure of $\mathfrak{S}_{n}$.

Proposition 3.4 The super-Lie representation $\mathfrak{S}_{n}$ and the twisted Lie representation $\varepsilon \mathrm{Lie}_{n}$ are isomorphic $\Sigma_{n}$-modules.

Proof The module $\mathrm{Lie}_{n}$ is spanned by all Lie monomials $g\left(x_{1}, x_{2}, \ldots, x_{n}\right)$ in which each of the $n$ generators occurs once. The $n$ variables occur in $g$ in a definite order: let this order, reading from left to right, be $x_{\gamma(1)}, x_{\gamma(2)}, \ldots, x_{\gamma(n)}$. This gives a permutation $\gamma \in \Sigma_{n}$ depending upon $g$. Every such $g$ is the bracket $[h, k]$ of two monomials in complementary subsets of the variables.

Let $\mathcal{J}$ be any Lie superring, and $y_{1}, y_{2}, \ldots, y_{n}$ any elements of odd degree in $\mathcal{J}$. We claim that there is a unique homomorphism $\Theta: \operatorname{Lie}_{n} \rightarrow \mathcal{J}$ of abelian groups such that for each monomial $g\left(x_{1}, x_{2}, \ldots, x_{n}\right)$ as above

$$
\Theta\left(g\left(x_{1}, x_{2}, \ldots, x_{n}\right)\right)=\varepsilon(\gamma) G\left(y_{1}, y_{2}, \ldots, y_{n}\right)
$$

where $G\left(y_{1}, y_{2}, \ldots, y_{n}\right)$ is the element of $\mathcal{J}$ obtained by replacing all the Lie bracket operators in $g$ by Lie superbrackets, then substituting $y_{i}$ for $x_{i}$; and $\varepsilon(\gamma)$ is the sign of $\gamma(g)$. The uniqueness of $\Theta$ is clear.

We construct $\Theta$ by induction on $n$. Suppose the result is known already for fewer than $n$ generators. Then if $g$ has $n$ variables and $g=[h, k]$ as above, we have $\Theta(g)=\eta \cdot[\Theta(h), \Theta(k)]$ where $[\Theta(h), \Theta(k)]$ is the superbracket and $\eta$ is the sign of the inverse shuffle which places the generators occurring in $h$ before those occurring in $k$. In order to show that $\Theta$ is a well-defined homomorphism in the 
$n$-variables case, we must verify that it respects the antisymmetry and Jacobi relations $[h, k]=-[k, h]$ and $[h,[k, l]]+[k,[l, h]]+[l,[h, k]]=0$ of $\operatorname{Lie}_{n}$. But this is true because the shuffle signs convert these relators into the corresponding signed relators, which are zero in the Lie superring $\mathcal{J}$. The induction is now complete.

It is not at all difficult to give an instance where $\Theta$ is a monomorphism: one begins by taking the $y_{i}$ to be elementary matrices representing elements of the endomorphism superring of a superspace of large rank. Therefore $\Theta$ is also a monomorphism when $\mathcal{J}$ is the free superring generated by the odd-degree elements $y_{1}, y_{2}, \ldots, y_{n}$. So in this case $\Theta$ is an isomorphism of abelian groups onto its image, which is $\mathfrak{S}_{n}$. For any $\sigma \in \Sigma_{n}$ we have

$$
\Theta(\sigma \cdot g)=\varepsilon(\sigma \gamma)(\sigma \cdot G)=\varepsilon(\sigma)(\sigma \cdot \Theta(g))
$$

Hence $\Theta$ induces an isomorphism between $\operatorname{Lie}_{n}$ and $\varepsilon \otimes \mathfrak{S}_{n}$, and between $\varepsilon \mathrm{Lie}_{n}$ and $\mathfrak{S}_{n}$.

The construction of the isomorphism between $\varepsilon \mathrm{Lie}_{n}$ and $\mathfrak{S}_{n}$ makes use of the natural ordering of the set $x_{1}, x_{2}, \ldots, x_{n}$ of generators. The effect of adopting a different fundamental ordering, related to the natural one by a permutation $\tau$, is to multiply the isomorphism by the sign of $\tau$. When the set of generators is unordered, the isomorphism is uniquely defined only up to sign.

Corollary 3.5 The left-regulated Lie superbrackets

$$
\lambda_{\sigma}=\left[x_{\sigma(1)},\left[x_{\sigma(2)},\left[\ldots\left[x_{\sigma(n-1)}, x_{n}\right] . .\right]\right]\right],
$$

where $\sigma \in \Sigma_{n-1}$, form a basis for $\mathfrak{S}_{n}$.

Proof This follows from 3.1 and 3.4 or by direct computation as for $\mathrm{Lie}_{n}$.

The first advantage of $\mathfrak{S}_{n}$ over $\varepsilon \mathrm{Lie}_{n}$ in our homology theory is that the Lie superbracket is extremely useful in building the fundamental orientation cycle. The second advantage is that the isomorphism of $\mathfrak{S}_{n}$ with the cohomology of $T_{n}$, which we shall construct in Theorem 4.1 below, is totally natural: it does not depend upon an ordering of the set of non-zero labels. 


\subsection{Construction of the fundamental cycle}

We now describe the construction of a certain cycle $F_{n}$ in the top-dimensional simplicial chain group $C_{n-3}\left(T_{n} ; \mathfrak{S}_{n}\right)$, which after 3.4 we know to be isomorphic to $C_{n-3}\left(T_{n} ; \varepsilon \mathrm{Lie}_{n}\right)$. A top-dimensional simplex $X$ of $T_{n}$ is an $n$-tree which is binary; that is, every internal node is the meet of three edges (see $\$ 2$ ). The vertices of the simplex correspond to the internal edges of the tree $X$; so an orientation of the simplex may be specified by a word $w_{X}=e_{0} e_{1} \ldots e_{n-3}$ in which each internal edge of $X$ appears once. We write this oriented simplex $\left\langle w_{X}\right\rangle$. If the order of the edges in $w_{X}$ is altered by a permutation $\sigma$, then $\left\langle w_{X}\right\rangle$ is multiplied by $\varepsilon(\sigma)$. We shall choose such an ordering for each $(n-3)$-simplex $X$ of $T_{n}$, and we shall set

$$
F_{n}=\sum_{X}\left\langle w_{X}\right\rangle \otimes c_{X} \quad \in \quad C_{n-3}\left(T_{n} ; \mathfrak{S}_{n}\right)
$$

where $c_{X} \in \mathfrak{S}_{n}$ is a coefficient monomial which we now describe.

We use induction on $n$ to define $w_{X}$ and $c_{X}$. When $n \leq 2$ the complex $T_{n}$ is empty, as the unique $n$-trees $X$ have no internal edges; but nevertheless we formally define $c_{X}=x_{1}$ when $n=1$ and $c_{X}=-\left[x_{1}, x_{2}\right]$ when $n=2$. Here, and throughout the rest of \$3 the bracket is the operation in a free Lie superring. The variables $x_{i}$ are in bijective correspondence with the non-zero tree labels. Since the $x_{i}$ are assigned odd grading, we have $\left[x_{1}, x_{2}\right]=\left[x_{2}, x_{1}\right]$. The total ordering on the set of labels has no significance.

We continue with our inductive definition. Suppose now that $n>2$.

The $n$-tree $X$ is obtained by grafting together at the root an $i$-tree $Y$ and an $(n-i)$-tree $Z$, where $0<i<n$, as in the following diagram

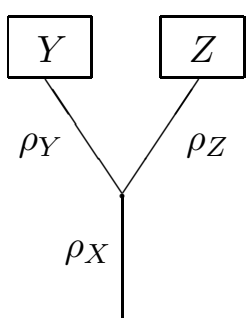

since the root of $X$ meets two other edges. The internal edges of $X$ comprise the internal edges of $Y$ and those of $Z$, together with the roots $\rho_{Y}$ and $\rho_{Z}$ of $Y$ and $Z$. Suppose by induction that we have well-defined terms $\left\langle w_{Y}\right\rangle \otimes c_{Y}$ and $\left\langle w_{Z}\right\rangle \otimes c_{Z}$ corresponding to orderings of the internal edges of $Y$ and $Z$, the 
coefficients $c_{Y}$ and $c_{Z}$ being super-Lie monomials in the sets of non-zero labels of $Y$ and $Z$ respectively. We set $w_{X}=\rho_{Y} w_{Y} \rho_{Z} w_{Z}$, this being an ordering of all the internal edges of $X$. The set of non-zero labels of $X$ is the disjoint union of those of $Y$ and $Z$. We put $c_{X}=(-1)^{|Y|}\left[c_{Y}, c_{Z}\right]$ where $|Y|$ is the number $i$ of leaves, excluding the root, in $Y$. This is a super-Lie monomial in the non-zero labels of $X$.

To justify this construction, we verify in the next two lemmas that $\left\langle w_{X}\right\rangle \otimes c_{X}$ is a well-defined element of the chain group $C_{n-3}\left(T_{n} ; \mathfrak{S}_{n}\right)$, and that the sum $F_{n}$ of these elements over all top-dimensional simplices $X$ is a cycle.

Lemma 3.7 The term

$$
\left\langle w_{X}\right\rangle \otimes c_{X} \quad \in \quad C_{n-3}\left(T_{n} ; \mathfrak{S}_{n}\right)
$$

is well defined for every binary $n$-tree $X$, and is $\Sigma_{n}$-invariant.

Proof We again use induction on $n$. For $n=1$ the result is completely trivial. When $n \geq 2$ the tree $X$ is obtained by grafting to the root an $i$-tree $Y$ and an $(n-i)$-tree $Z$, where $0<i<n$ and therefore both $\left\langle w_{Y}\right\rangle \otimes c_{Y}$ and $\left\langle w_{Z}\right\rangle \otimes c_{Z}$ are well-defined by inductive hypothesis.

Since the oriented simplices $\left\langle w_{Y}\right\rangle$ and $\left\langle w_{Z}\right\rangle$ are evidently well defined up to sign, the coefficients $c_{Y}$ and $c_{Z}$ are well defined up to sign. Therefore the total sign in the formula

$$
\left\langle w_{X}\right\rangle \otimes c_{X}=\left\langle\rho_{Y} w_{Y} \rho_{Z} w_{Z}\right\rangle \otimes(-1)^{|Y|}\left[c_{Y}, c_{Z}\right]
$$

is independent of the orientations of the two simplices $Y$ and $Z$, by bilinearity of tensor product. The only remaining ambiguity is that the sub-trees $Y$ and $Z$ can be listed in either order. Interchanging them may affect the orientation of the simplex $X$, and may alter the sign of $c_{X}=(-1)^{|Y|}\left[c_{Y}, c_{Z}\right]$.

We compute the change in the total sign when $Y$ and $Z$ are interchanged. Since the number of internal edges in a binary tree equals the number of leaves (excluding the root) less two, the effect of interchanging $\rho_{Y} w_{Y}$ and $\rho_{Z} w_{Z}$ is to multiply $\left\langle w_{X}\right\rangle$ by the sign $(-1)^{(i-1)(n-i-1)}$. On the other hand, the replacement of $(-1)^{|Y|}$ by $(-1)^{|Z|}$ introduces a sign $(-1)^{n}$, whilst the replacement of the superbracket $\left[c_{Y}, c_{Z}\right]$ by $\left[c_{Z}, c_{Y}\right]$ gives a factor $(-1)^{i(n-i)+1}$, because $c_{Y}$ and $c_{Z}$ have degrees $i$ and $n-i$ respectively. The product of these four signs is +1 , so that $\left\langle w_{X}\right\rangle \otimes c_{X}$ is independent of the order in which $Y$ and $Z$ are taken. The inductive step is thus complete. Finally, the $\Sigma_{n}$-invariance of $\left\langle w_{X}\right\rangle \otimes c_{X}$ is obvious, since the ordering of the set of labels has nowhere been used. The lemma is proved. 
Proposition 3.8 The $(n-3)$-chain

$$
F_{n}=\sum_{X}\left\langle w_{X}\right\rangle \otimes c_{X} \quad \in \quad C_{n-3}\left(T_{n} ; \mathfrak{S}_{n}\right)
$$

is a $\Sigma_{n}$-invariant cycle.

Proof The $\Sigma_{n}$-invariance of $F_{n}$ follows from Lemma 3.7 .

To prove that $F_{n}$ is a cycle, we calculate all the coefficients of the $(n-4)$-chain $\partial F_{n}$. Every oriented $(n-4)$-simplex $\sigma$ corresponds to a tree $S$ which is binary apart from one exceptional node, where four edges meet. There are two cases, depending upon the position of the exceptional node.

Suppose first that the exceptional node is the root node. Then $S$ is the result of grafting together three trees $X, Y$ and $Z$, as shown on the left here. As orientation for $\sigma$ we may take the edge-sequence $\left\langle\rho_{X} w_{X} \rho_{Y} w_{Y} \rho_{Z} w_{Z}\right\rangle$, since this lists all the internal edges (in the notation of [3.6). One of the three $(n-3)$ simplices incident upon $\sigma$ is obtained by grafting $X$ to $Y$ (which introduces a new root $\rho_{X Y}$ ) and grafting the result to $Z$, as in the picture on the right.
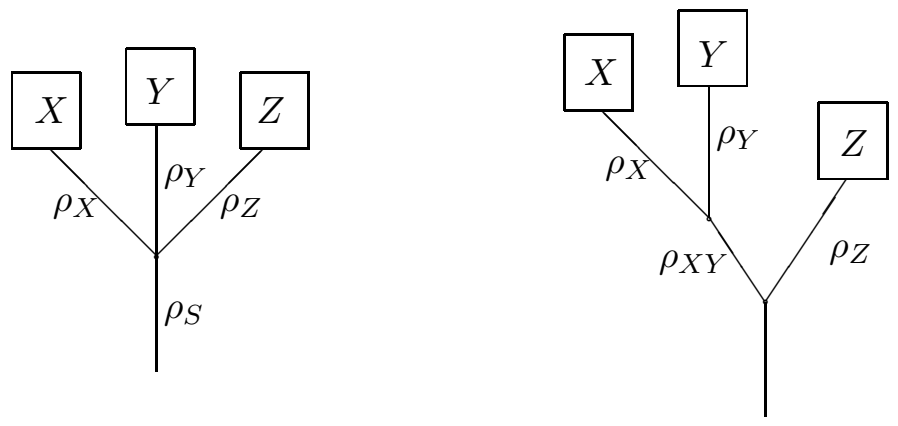

This simplex can be oriented as $\left\langle\rho_{X Y} \rho_{X} w_{X} \rho_{Y} w_{Y} \rho_{Z} w_{Z}\right\rangle$ : then its incidence index upon $\sigma$ is +1 , and its contribution to the coefficient of $\partial F_{n}$ on $\sigma$ is, by two applications of the construction in 3.6

$$
(-1)^{|Y|}\left[\left[c_{X}, c_{Y}\right], c_{Z}\right]
$$

The other two $(n-3)$-simplices incident on $\sigma$ are obtained from the first by permuting $X, Y$ and $Z$ cyclically. The simplex $\left\langle\rho_{Y Z} \rho_{Y} w_{Y} \rho_{Z} w_{Z} \rho_{X} w_{X}\right\rangle$ has incidence index $(-1)^{(|X|+1)(|Y|+|Z|)}$ upon $\sigma$ (oriented as before), and the applications of the construction of 3.6 contribute altogether a sign $(-1)^{|Z|}$. The second simplex therefore contributes

$$
(-1)^{|Y|+|X||Y|+|X||Z|}\left[\left[c_{Y}, c_{Z}\right], c_{X}\right] .
$$


By cyclic permutation, the third simplex incident upon $\sigma$ contributes

$$
(-1)^{|Y|+|Z||X|+|Z||Y|}\left[\left[c_{Z}, c_{X}\right], c_{Y}\right] .
$$

The total coefficient of $\partial F_{n}$ on $\sigma$ is thus zero in view of the Jacobi identity

$$
(-1)^{|Y||Z|}\left[\left[c_{Z}, c_{X}\right], c_{Y}\right]+(-1)^{|Z||X|}\left[\left[c_{X}, c_{Y}\right], c_{Z}\right]+(-1)^{|X||Y|}\left[\left[c_{Y}, c_{Z}\right], c_{X}\right]=0
$$

in the Lie superring. The first case is proved.

In the second case, the exceptional node (where four edges meet) of $S$ is not the root node. Thus $S$ is obtained by grafting together at the root a binary tree $T$ and a tree $U$ having one exceptional node. Let $m$ be the number $|U|$ of leaves of $U$. It follows by application of the definition in 3.6 that the coefficient of $\partial F_{n}$ on $\sigma$ is $\left[c_{T}, e\right]$ where $e$ is the coefficient of $\partial F_{m}$ on an appropriate orientation of the simplex represented by $U$. By induction on $n$, we know that $F_{m}$ is a cycle, so $e$ is zero. This establishes the second case, and completes the proof of 3.8 .

\section{Example 3.9}

We illustrate the foregoing theory by calculating the fundamental cycle $F_{5}$ of $T_{5}$. To reinforce the point that the ordering of the tree labels is nowhere used, we label the trees by $\{0, a, b, c, d, e\}$ in place of $\{0,1,2,3,4,5\}$. We use $a, b, c, d, e$ also to denote the corresponding odd-degree generators of the Lie superring.

We enumerate the top-dimensional simplices of $T_{5}$. There are 105 of these, generated by the trees pictured here and the action of the symmetric group $\Sigma_{5}$ on the labels $a, b, c, d, e$. We choose orientations for the simplices by ordering the internal edges, and then calculate the 105 corresponding terms in the cycle $F_{5}$. Each term is obtained by using the formula of 3.6 twice.

There are 60 terms of the form $\Phi=\langle x, y, z\rangle \otimes[a,[b,[c,[d, e]]]]$ corresponding to trees of the shape:

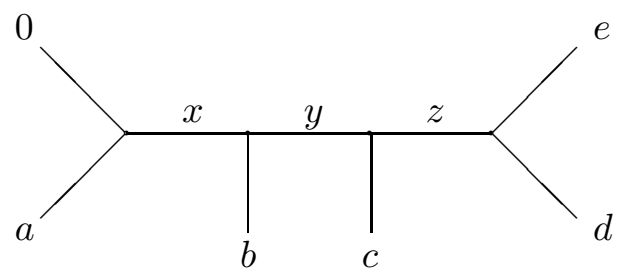


another 30 terms of the form $\Psi=-\langle p, q, r\rangle \otimes[[a, b],[c,[d, e]]]$ corresponding to trees of the shape:

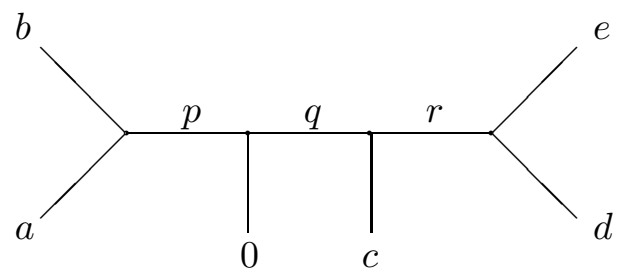

and 15 terms of the form $\Omega=-\langle i, j, k\rangle \otimes[a,[[b, c],[d, e]]]$ corresponding to trees of the shape:

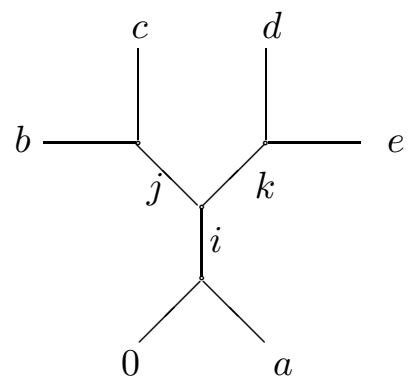

The number of terms in each case is given by dividing $5 !=120$ by the order of the symmetry group of the tree fixing the root. Our construction takes care of the signs. We have

$$
\begin{gathered}
F_{5}=\sum\langle x, y, z\rangle \otimes[a,[b,[c,[d, e]]]]-\sum\langle p, q, r\rangle \otimes[[a, b],[c,[d, e]]] \\
-\sum\langle i, j, k\rangle \otimes[a,[[b, c],[d, e]]]
\end{gathered}
$$

where there are 105 terms in all, corresponding to all the labelled trees up to label-preserving isomorphism. (The letters attached to the internal edges are bound variables over which summation is carried out: other symbols can be substituted for them at will.) Now we check that the boundary $\partial F_{5}$ is zero. We revert to the notation $\Phi, \Psi, \Omega$ for the three types of terms in $F_{5}$. On each type there are three face operators to consider.

First we look at the terms $\partial_{2} \Phi$. We have $\partial_{2} \Phi=\langle x, y\rangle \otimes[[a, b],[c,[d, e]]]$ where $\langle x, y\rangle$ is an orientation of the tree: 


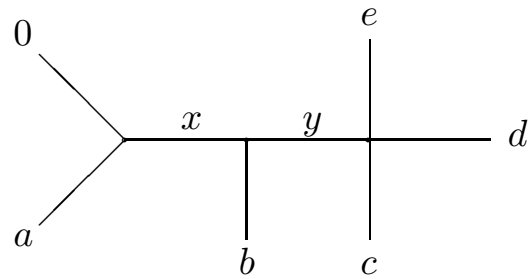

in which the leaves $c, d$ and $e$ are attached at the same node. Therefore the 60 terms $\partial_{2} \Phi$ cancel among themselves in threes by virtue of the relation

$$
[c,[d, e]]+[d,[e, c]]+[e,[c, d]]=0
$$

in the coefficient module $\mathfrak{S}_{5}$. In the same way, the 30 terms $\partial_{2} \Psi$ have sum zero, and the 60 terms $\partial_{0} \Phi$ cancel the 30 terms $\partial_{0} \Psi$ by the supercommutator formula

$$
[[a, b],[c,[d, e]]]-[a,[b,[c,[d, e]]]]-[b,[a,[c,[d, e]]]]=0 .
$$

Finally each of the 15 terms $\partial_{0} \Omega$ cancels with a pair of terms $\partial_{1} \Psi$ through

$$
[a,[[b, c],[d, e]]]=[[b, c],[a,[d, e]]]-[[d, e],[a,[b, c]]]
$$

and each of the terms in $\partial_{1} \Omega$ or $\partial_{2} \Omega$ likewise cancels a pair of terms in $\partial_{1} \Phi$. This accounts for all 315 terms in $\partial F_{5}$, and verifies that $F_{5}$ is a cycle.

\section{The homology and cohomology as integral repre- sentations of $\Sigma_{n}$}

We showed in $\S 2$ that the space $T_{n}$ has reduced homology only in dimension $n-3$. We now determine the action of $\Sigma_{n}$ on this finite $\mathbb{Z}$-module. The following theorem is equivalent to a result proved by M. Wachs [12 for the cohomology of the poset of partitions.

Theorem 4.1 There are isomorphisms of left $\Sigma_{n}$-modules and of right $\Sigma_{n^{-}}$ modules respectively

$$
\begin{aligned}
\tilde{H}^{*}\left(T_{n} ; \mathbb{Z}\right) & \approx \mathfrak{S}_{n} \approx \varepsilon \operatorname{Lie}_{n} \\
\tilde{H}_{*}\left(T_{n} ; \mathbb{Z}\right) & \approx \mathfrak{S}_{n}^{*} \approx \varepsilon \operatorname{Lie}_{n}^{*} .
\end{aligned}
$$


Proof As $T_{n}$ is a finite complex with torsion-free homology, there is for every coefficient group $G$ a natural universal coefficient isomorphism

$$
\tilde{H}_{*}\left(T_{n} ; G\right) \approx \operatorname{Hom}_{\mathbb{Z}}\left(\tilde{H}^{*}\left(T_{n}\right), G\right) .
$$

In particular, the integral homology and integral cohomology are dual $\mathbb{Z}$-modules. In the above we take $G$ to be $\mathfrak{S}_{n}$ or the isomorphic module $\varepsilon \mathrm{Lie}_{n}$. The homology class of the invariant cycle $F_{n}$ constructed in 3.6 corresponds under the universal coefficient isomorphism to some homomorphism $\theta_{n}: \tilde{H}^{*}\left(T_{n}\right) \rightarrow \mathfrak{S}_{n}$, which is indeed $\Sigma_{n}$-equivariant because the universal coefficient isomorphism is natural and $F_{n}$ is $\Sigma_{n}$-invariant.

Let $f_{\sigma}$ be the cocycle of $T_{n}$ which takes the value 1 on the simplex $\gamma_{\sigma}$ depicted in 2.5. and is zero on all other $(n-3)$-simplices of $T_{n}$. Then the set

$$
\left\{\left[f_{\sigma}\right] \mid \sigma \in \Sigma_{n-1}\right\}
$$

is a basis for $\tilde{H}^{*}\left(T_{n}\right)$, by 2.5. The value of $\theta_{n}$ on $\left[f_{\sigma}\right]$ is $\pm \lambda_{\sigma}$, by 3.6] and induction on $n$, where $\lambda_{\sigma}$ is the basis element $\left[x_{\sigma(1)},\left[x_{\sigma(2)},\left[\ldots\left[x_{\sigma(n-1)}, x_{n}\right] ..\right]\right]\right]$ of the $\mathbb{Z}$-module $\mathfrak{S}_{n}$, as in 3.5 . Therefore we have shown that the $\Sigma_{n}$-homomorphism $\theta_{n}$ carries a basis for $\tilde{H}^{*}\left(T_{n}\right)$ into a basis for $\mathfrak{S}_{n}$, which is isomorphic to $\varepsilon \mathrm{Lie}_{n}$ by 3.4. That proves the first assertion in the theorem. The second assertion follows by taking $\mathbb{Z}$-duals.

Corollary 4.2 The $\Sigma_{n}$-module $\mathrm{Lie}_{n}$ is the restriction of a left $\Sigma_{n+1}$-module.

Proof We have seen that $\Sigma_{n+1}$ acts on $T_{n}$, so it acts upon $\varepsilon \tilde{H}^{*}\left(T_{n}\right)$, which is isomorphic to $\operatorname{Lie}_{n}$.

Definition 4.3 We denote by $\widehat{\operatorname{Lie}}_{n}$ the left $\Sigma_{n+1}$-module so defined, and by $\widehat{\operatorname{Lie}}_{n}^{*}$ the dual right $\Sigma_{n+1}$-module. Evidently $\operatorname{Res}_{\Sigma_{n}}^{\Sigma_{n+1}} \widehat{\operatorname{Lie}}_{n} \approx \operatorname{Lie}_{n}$.

\section{The Whitehouse extension}

Let $H$ be a subgroup of the finite group $G$. Since the functor $\operatorname{Ind}_{H}^{G}$ is left adjoint to $\operatorname{Res}_{H}^{G}$, there is an adjunction morphism $\operatorname{Ind}_{H}^{G} \operatorname{Res}_{H}^{G} M \longrightarrow M$ for every $G$-module $M$, and it is always surjective.

The following theorem was proved algebraically by Whitehouse [13]; it also follows from work of Sundaram [10]. 
Theorem 5.1 There is a short exact sequence of $\Sigma_{n+1}$-modules

$$
0 \longrightarrow \operatorname{Lie}_{n+1}^{*} \longrightarrow \operatorname{Ind}_{\Sigma_{n}}^{\Sigma_{n+1}} \operatorname{Lie}_{n}^{*} \longrightarrow \widehat{\operatorname{Lie}}_{n}^{*} \longrightarrow 0
$$

in which the surjection is the adjunction morphism connecting $\operatorname{Ind}_{\Sigma_{n}}^{\Sigma_{n+1}}$ and $\operatorname{Res}_{\Sigma_{n}}^{\Sigma_{n+1}}$.

We shall give a topological proof. We recall that there is an analogue for $G$ spaces of the induction functor $\operatorname{Ind}_{H}^{G}$, namely smash product with $(G / H)_{+}$. Theorem 5.1 follows immediately from the cofibration in the following theorem, by taking reduced homology.

Theorem 5.2 There is a $\Sigma_{n+1}$-equivariant cofibration

$$
X \subset T_{n+1} \longrightarrow\left(\Sigma_{n+1} / \Sigma_{n}\right)_{+} \wedge S T_{n}
$$

where $X$ is $\Sigma_{n+1}$-homotopy equivalent to $T_{n}$ and $S$ denotes suspension.

Proof In the tree space $T_{n+1}$ we consider for each $1 \leq i \leq n+1$ the star $\operatorname{st}\left(v_{0 i}\right)$ of the vertex $v_{0 i}$ of 2.2. This consists of all the $(n+1)$-trees in which the leaves labelled 0 and $i$ are separated from the rest of the tree by an internal edge of positive length. These $n+1$ open stars are therefore disjoint. The closure $\overline{\text { st }}\left(v_{0 i}\right)$ is isomorphic to the contractible space $\tilde{T}_{n}$, by the homeomorphism which deletes the leaf 0 and then relabels the leaf $n+1$ as 0 . Let $X$ be the complement $T_{n+1} \backslash \bigcup_{i=1}^{n+1} \operatorname{st}\left(v_{0 i}\right)$. By 2.3 the subcomplex $\tilde{T}_{n}$ is the cone on $T_{n}$, so

$$
T_{n+1} / X \approx \bigvee_{i=1}^{n+1} \overline{\operatorname{st}}\left(v_{0 i}\right) / \partial\left(\overline{\operatorname{st}}\left(v_{0 i}\right)\right) \approx \bigvee_{i=1}^{n+1} S T_{n} .
$$

The isomorphism $\overline{\operatorname{st}}\left(v_{0, n+1}\right) / \partial\left(\overline{\operatorname{st}}\left(v_{0, n+1}\right)\right) \approx S T_{n}$ is $\Sigma_{n}$-equivariant, and $\Sigma_{n+1}$ induces a bijection between the summands and the left cosets $\Sigma_{n+1} / \Sigma_{n}$, so the wedge on the right above is equivariantly isomorphic to $\left(\Sigma_{n+1} / \Sigma_{n}\right)_{+} \wedge S T_{n}$.

We must now prove that $X$ is $\Sigma_{n+1}$-homotopy-equivalent to $T_{n}$, where $\Sigma_{n+1}$ acts on $T_{n}$ by permuting the labels $\{0,1, \ldots, n\}$, with 0 in place of $n+1$. We assume $n \geq 3$ : the reasoning below requires trivial modifications for $n=1,2$.

Given any tree in $T_{n}$, we obtain a tree in $X \subset T_{n+1}$ by the following procedure: relabel the leaf 0 as $n+1$, then attach a new leaf labelled 0 at any point on any internal edge. Every point of $X$ arises once only in this way, so the reverse procedure (deleting the leaf 0 and relabelling the leaf $n+1$ as 0 ) gives a projection $\varphi: X \rightarrow T_{n}$. As usual, when the leaf-deletion results in the 
amalgamation of two edges with lengths $x$ and $y$, the new combined edge is assigned length $\max (x, y)$.

The inverse image under $\varphi$ of any point $t \in T_{n}$ is isomorphic to the subtree consisting of the internal edges of $t$. This is contractible. By the Vietoris Theorem 5.3 below, $\varphi$ is a homotopy equivalence.

The map $\varphi$ is $\Sigma_{n+1}$-equivariant by construction. To show that it is a equivariant homotopy equivalence, it suffices to show that, for every subgroup $H$ of $\Sigma_{n+1}$, the restriction $\varphi^{H}: X^{H} \rightarrow T_{n}^{H}$ between the fixed point sets is a homotopy equivalence. If $t \in T_{n}^{H}$ is any $H$-fixed point, then the group $H$ acts by isometries on the tree $t$, permuting the labels.

Whenever a finite group acts by isometries on a finite tree, the set of invariant points is contractible. In our case the invariant points form the inverse image of the point $t$ under $\varphi^{H}$. Thus $\varphi^{H}$ satisfies the hypotheses of Theorem [5.3. and the induced map of fixed point sets is always a homotopy equivalence, which proves Theorem 5.2 and therefore Theorem 5.1

The Vietoris theorem used in the above proof was proved in the following strong form by M. M. Cohen ([5], Theorem 11.1).

Theorem 5.3 A piecewise linear map between finite polyhedra is a simple homotopy equivalence if the inverse image of every point is contractible.

Homotopy equivalence under these hypotheses is a theorem of Smale [9]. The simply connected case already follows from Vietoris [11] by an application of Whitehead's Theorem.

\section{Acknowledgements}

The author acknowledges the benefit of conversations with Teimuraz Pirashvili, Colin Rourke and Brian Sanderson, and valuable commentary from the referee.

\section{References}

[1] J M Boardman, Homotopy structures and the language of trees, Algebraic topology (Wisconsin, 1970) Proc. Symp. Pure Math. 22 (1971) 37-58 MathReview

[2] J M Boardman, R M Vogt, Homotopy invariant algebraic structures on topological spaces, Lect. Notes in Math. 347 Springer-Verlag (1973) MathReview 
[3] $\quad$ F R Cohen, The homology of $\mathcal{C}_{n+1}$-spaces, $n \geq 0$, from: "The homology of iterated loop spaces", Lect. Notes in Math. 533, Springer-Verlag (1976) 207-351 MathReview

[4] F R Cohen, On configuration spaces, their homology, and Lie algebras, J. Pure App. Algebra 100 (1995) 19-42 MathReview

[5] M M Cohen, Simplicial structures and transverse cellularity, Ann. Math. 85 (1967) 218-245 MathReview

[6] B Fresse, Koszul duality of operads and homology of partition posets, from: "Homotopy theory: relations with algebraic geometry, group cohomology and $K$-theory (Evanston 2002)", Contemp. Math. 346 (2004) 115-215 MathReview

[7] C Reutenauer, Free Lie algebras, London Mathematical Society Monographs 7, Oxford University Press (1993) MathReview

[8] A Robinson, S Whitehouse, The tree representation of $\Sigma_{n+1}$, J. Pure Appl. Algebra 111 (1996) 245-253 MathReview

[9] S Smale, A Vietoris mapping theorem for homotopy, Proc. Amer. Math. Soc. 8 (1957) 604-610 MathReview

[10] S Sundaram, Homotopy of non-modular partitions and the Whitehouse module, J. Algebraic Combin. 9 (1999) 251-269 MathReview

[11] L Vietoris, Über den höheren Zusammenhang kompacter Räume und eine Klasse von zusammenhangstreuen Abbildungen, Math. Ann. 97 (1927) 454-472

[12] ML Wachs, On the (co)homology of the partition lattice and the free Lie algebra, from: "Selected papers in honor of Adriano Garsia (Taormina, 1994)", Discrete Mathematics 193 (1998) 287-319 MathReview

[13] S Whitehouse, The integral tree representation of the symmetric group, J. Algebraic Combin. 13 (2001) 317-326 MathReview

Mathematics Institute, University of Warwick, Coventry CV4 7AL, UK

Email: car@maths.warwick.ac.uk

Received: 17 February 2004 